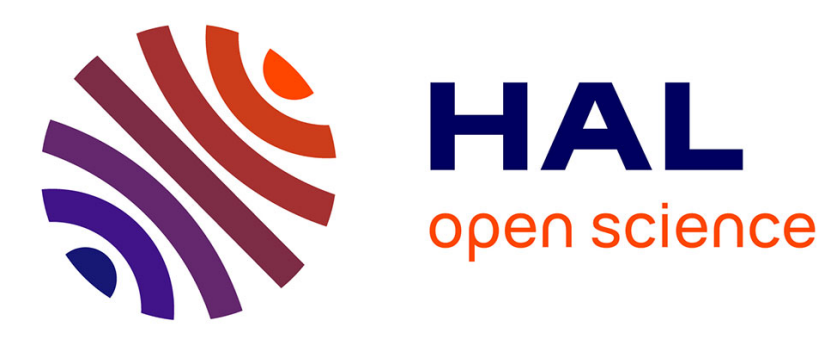

\title{
Temporal Filtering of Line Scratch Detections in Degraded Films
}

\author{
Alasdair Newson, Andrés Almansa, Yann Gousseau, Patrick Pérez
}

\section{To cite this version:}

Alasdair Newson, Andrés Almansa, Yann Gousseau, Patrick Pérez. Temporal Filtering of Line Scratch Detections in Degraded Films. International Conference on Image Processing (ICIP), IEEE, Sep 2013, Melbourne, Australia. pp.4088-4092, 10.1109/ICIP.2013.6738842 . hal-00927487

\section{HAL Id: hal-00927487 https://hal.science/hal-00927487}

Submitted on 11 Sep 2018

HAL is a multi-disciplinary open access archive for the deposit and dissemination of scientific research documents, whether they are published or not. The documents may come from teaching and research institutions in France or abroad, or from public or private research centers.
L'archive ouverte pluridisciplinaire HAL, est destinée au dépôt et à la diffusion de documents scientifiques de niveau recherche, publiés ou non, émanant des établissements d'enseignement et de recherche français ou étrangers, des laboratoires publics ou privés. 


\title{
TEMPORAL FILTERING OF LINE SCRATCH DETECTIONS IN DEGRADED FILMS
}

\author{
Alasdair Newson ${ }^{\star \dagger} \quad$ Andrés Almansa ${ }^{\dagger} \quad$ Yann Gousseau ${ }^{\dagger} \quad$ Patrick Pérez $^{\star}$ \\ * Technicolor, 975 Avenue des Champs Blancs, 35570 Cesson-Sévigné, France \\ †Télécom ParisTech, CNRS LTCI 46 Rue Barrault, 75013 Paris, France
}

\begin{abstract}
The film defect known as the line scratch is difficult to restore automatically due to the large number of false alarms present in scratch detection algorithms. In this paper, an algorithm for dealing with these false alarms is proposed. Validating true scratches, which is the approach generally proposed in the literature, is a difficult task since scratch characteristics are hard to determine, making tracking these defects problematic. Instead, we eliminate false alarms by analysing their compatibility with a global motion estimation. We compare our algorithm with two other scratch detection methods from the literature. Experiments show that our algorithm outperforms these two, and that the proposed temporal filtering greatly improves precision while maintaining high recall.
\end{abstract}

Index Terms - Film defects, line scratches, affine dominant motion, temporal filtering

\section{INTRODUCTION}

Recent high-profile projects, such as the restoration of George Melies's "Voyage dans la Lune" (1902) ${ }^{1}$ reflect interest in the subject of digital film restoration. The digitalisation and transfer of old films into high definition formats implies that high quality restoration is required.

Common defects in films include dust/dirt, blotches, flicker and line scratches. In this paper, we concentrate on the last of these defects, the line scratch. Line scratches present certain singular characteristics, such as temporal persistence, which require special treatment. Scratches are usually detected by identifying straight, vertical lines which are darker or brighter than their surroundings $[1,2]$. In this paper we present a method to deal with the difficult problem of false alarms due to the line scratch detection process. These false alarms are mostly due to thin vertical scene structures which closely resemble line scratches. Contrary to previous work $[3,4]$, rather than try to validate true scratches, whose characteristics are difficult to determine, we explicitly reject false alarms. Using dominant affine motion estimation, we transform the problem of determining the false alarms' trajectories into the simpler problem of detecting straight lines in a binary

\footnotetext{
${ }^{1}$ Partly restored by Technicolor, presented at the Cannes Film Festival 2011.
}

detection map. Once these trajectories are detected, the false alarms belonging to the trajectories may be eliminated.

The outlay of the paper is as follows. In Section 2 we briefly recall previous work. In Section 3, we present a new motion estimation-based algorithm for temporal filtering of spatial detections in order to reject false alarms. Finally, experimental validations are presented in Section 4 on a series of degraded film sequences in comparison with previous work.

\section{PRIOR WORK}

Line scratch detection algorithms can be divided into two categories: spatial and temporal. Although we concentrate here on temporal filtering, we shall mention a few spatial algorithms, which are necessary for any temporal algorithm.

The first work concerning line scratch detection was carried out by Kokaram [1], and introduced a scratch model which is widely used in other papers (e.g., [5] and [6]). The Hough transform is used in both [1] and [7] to detect prominent lines. Recently Newson et al. [8] presented an algorithm which uses a contrario methods [9] for the robust detection line scratches. This algorithm has the advantage of describing the beginning and end points of a scratch precisely. In the present method, we will build upon the work in [8].

Temporal approaches may be found in [10], [11], [3] and [4]. The main goal of these temporal algorithms is to validate scratches using certain temporal hypotheses. In the first papers, Joyeux et al. rely on a sinusoidal hypothesis concerning scratch motion. A more recent method by Gullu et al. which exploits temporal information may be found in [4]. The authors use the local matching error between frames to determine if a detection is a true scratch or a vertical structure. The main limitation of these approaches is that robust and generic hypotheses concerning the spatio-temporal nature of true scratches are difficult to define.

\section{TEMPORAL FILTERING ALGORITHM}

All spatial line scratch detection algorithms suffer from false alarms due to thin vertical structures which closely resemble line scratches. In some situations, it is practically impossible to differentiate the two without prior knowledge concerning the scene structure. Unfortunately this sort of knowledge is 


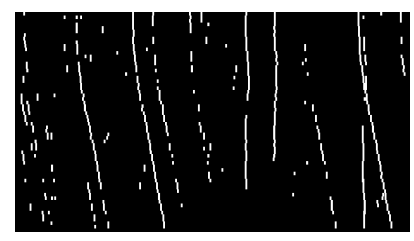

(a) Original detections $\left(I_{T}\right)$

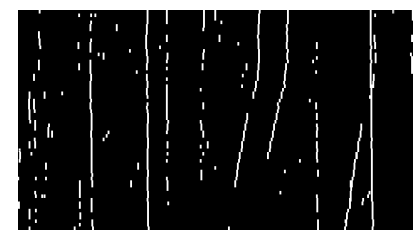

(b) Realigned detections $\left(I_{T}^{\prime}\right)$

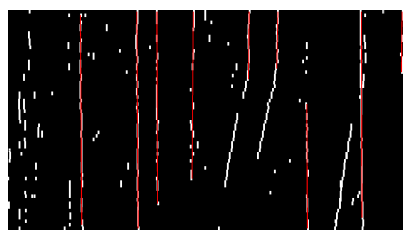

(c) Potential false detection trajectories in red

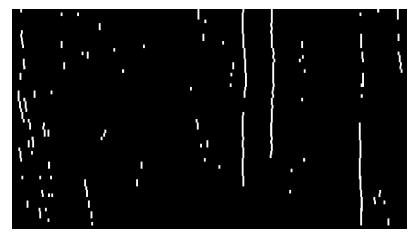

(d) Final filtered detections

Fig. 1. Different $x$-t binary maps, for the "Afgrunden 2" sequence. The $x$ axis represents the average column of a scratch, and the $y$ axis represents the frame number $(t)$ which the scratch belongs to. Each white point corresponds to a detected scratch. The original video may be viewed at : http://www.enst.fr/ gousseau/scratchfiltering.

difficult to obtain and use. Another possibility is to use the temporal information in the image sequence. This information is useful since the physical motion of a scratch is independent of that of the scene and therefore will differ in general.

The goal of our approach is the rejection of as many false alarms as possible rather than the validation of true scratches as in [3,4]. False alarms exhibit temporal characteristics which are easier to identify than those of true scratches.

False alarms due to vertical scene structures will naturally display similar motion to the underlying scene, since they are part of it. Therefore, we reject any scratch detections which follow a trajectory which conforms to the dominant scene motion. We refer to this hypothesis as the motion coherency hypothesis. Further simple hypotheses may be used for filtering false detections. One example, which we shall use, is a scene cut hypothesis, which stipulates that any trajectories start or stop close to a scene cut must correspond to false alarms.

These criteria do not deal with scratches which move with the scene, stop at a scene cut, or a completely still scene with static scratches. However, such situations are impossible to resolve without prior knowledge on the nature of scratches.

\subsection{Motion coherency}

In order to exploit the motion coherency hypothesis, we must determine the trajectories of the detected line scratches. In the present case, rather than trying to solve the exceedingly difficult task of correctly tracking objects such as scratches (as in [3]), we shall realign the spatial detections with respect to the motion of the scene. If this is done correctly, the false detections due to vertical scene structures should appear as straight lines in a spatio-temporal representation (on an $\mathrm{x}-\mathrm{t}$ axis as may be seen in Figure 1 (b)), transforming the problem into one of detecting vertical lines in a binary image. This problem is much more constrained than that of tracking true scratches, and therefore easier to solve.

We suppose that the line scratches have previously been detected using a spatial detection algorithm. The detections of this algorithm should represent the scratches as line segments in an image. In our experiments, we use our previous work of
[8] to carry out this spatial detection.

Let $I_{T}$ be the x-t binary detection map of the spatially detected scratches. In this detection map, each scratch detection is represented by its average column position (on the $x$-axis) and the index of the frame in which it is found (on the $y$-axis). An example of this representation may be seen in Figure 1 (a).

Let us introduce some notation. Let $S$ represent a scratch detection. We will refer to this as a segment. Let $\tilde{x}(S)$ and $\tilde{y}(S)$ be the average column and row indices of the segment, and $t(S)$ denote the frame in which segment was detected.

We wish to realign the scratches with the global scene motion. We recall that the motivating idea behind this realignment is to make the false detections appear as straight lines in an $\mathrm{x}$-t representation, and thus make their trajectories easier to determine.

In our work, we use the algorithm of Odobez et al. [12] to estimate a dominant affine motion. At a pixel position $q=$ $\left(x_{q}, y_{q}\right)$, the motion vector $(u(q), v(q))$ is expressed as :

$$
\left\{\begin{array}{l}
u(q)=c_{1}+a_{1} x_{q}+a_{2} y_{q} \\
v(q)=c_{2}+a_{3} x_{q}+a_{4} y_{q}
\end{array}\right.
$$

where $c_{1}$ and $c_{2}$ are the parameters describing the constant motion components, and $a_{1} \ldots a_{4}$ are the parameters associated with the spatially varying components of the motion.

If $(x, y)$ is a pixel in a frame $t$ and $\left(x^{\prime}, y^{\prime}\right)$ is the corresponding position in frame $t+1$, we have the following relationship :

$$
\left[\begin{array}{c}
x^{\prime} \\
y^{\prime} \\
1
\end{array}\right]=\left[\begin{array}{ccc}
a_{1}+1 & a_{2} & c_{1} \\
a_{3} & a_{4}+1 & c_{2} \\
0 & 0 & 1
\end{array}\right]\left[\begin{array}{l}
x \\
y \\
1
\end{array}\right]:=\tilde{A}_{t, t+1}\left[\begin{array}{l}
x \\
y \\
1
\end{array}\right] .
$$

The estimation is carried out between each consecutive pair of frames. For any $x, y$ and frame $t$ we can find the corresponding coordinates $\left(x^{r}, y^{r}\right)$ in the reference frame $r$, with the following relationship :

$$
\left[\begin{array}{c}
x^{r} \\
y^{r} \\
1
\end{array}\right]=\tilde{A}_{r, t}^{-1}\left[\begin{array}{l}
x \\
y \\
1
\end{array}\right]
$$




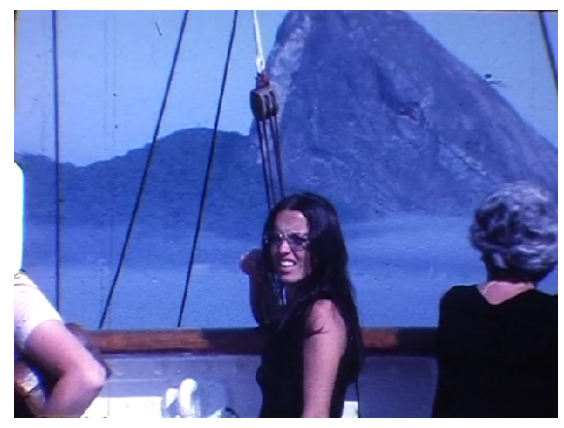

(a) Original frame

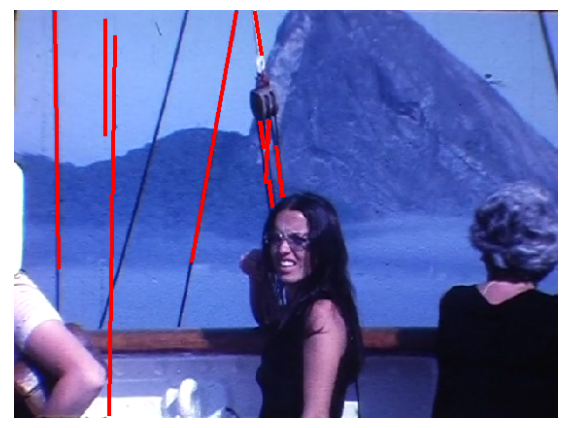

(b) Spatial detections (in red as in [8])

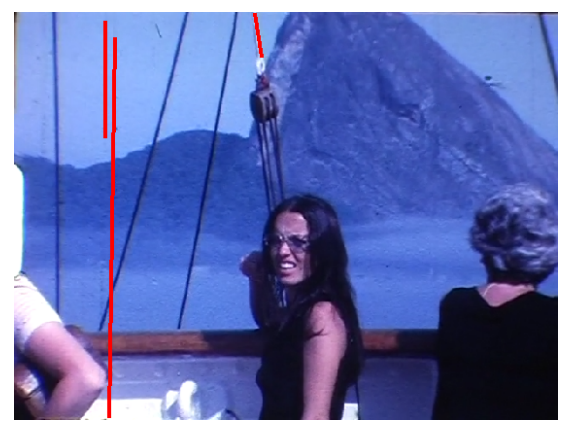

(c) Detections after temporal filtering by the proposed method

Fig. 2. Detections from a frame of "Keldjian" with false alarms due to thin vertical objects removed with temporal filtering.

\begin{tabular}{|c|c|c|c|c|c|c|c|c|c|c|c|c|}
\hline \multirow{2}{*}{ Film } & \multicolumn{4}{|c|}{ Recall (\%) } & \multicolumn{4}{|c|}{ Precision (\%) } & \multicolumn{4}{|c|}{$f 1$-score $(\%)$} \\
\hline & Bruni [2] & Gullu [4] & Spatial [8] & Proposed & Bruni & Gullu & Spatial & Proposed & Bruni & Gullu & Spatial & Proposed \\
\hline Knight & 100.00 & 100.00 & 79.34 & 79.34 & 29.54 & 29.54 & 71.70 & 71.70 & 45.61 & 45.61 & 75.33 & 75.33 \\
\hline Sitdown & 80.93 & 80.93 & 74.72 & 74.72 & 56.47 & 56.47 & 72.24 & 72.24 & 66.52 & 66.52 & 73.46 & 73.46 \\
\hline Star & 95.00 & 95.00 & 85.28 & 82.67 & 56.87 & $\mathbf{5 7 . 3 2}$ & 53.84 & 56.95 & 71.15 & 71.50 & 66.01 & 67.44 \\
\hline Afgrunden 1 & 75.11 & 68.75 & 87.63 & 87.63 & 09.35 & 10.27 & 46.00 & 66.89 & 16.63 & 17.86 & 60.33 & 75.87 \\
\hline Afgrunden 2 & 57.72 & 52.98 & 94.33 & 94.01 & 07.85 & 07.43 & 28.02 & 49.80 & 13.82 & 13.03 & 43.21 & 65.11 \\
\hline Keldjian & 12.63 & 11.48 & 78.46 & 78.28 & 07.11 & 07.63 & 17.66 & 37.99 & 09.10 & 09.17 & 28.83 & 51.15 \\
\hline Afgrunden gate & 49.81 & 31.29 & 89.09 & 89.09 & 02.45 & 02.28 & 03.32 & 11.88 & 04.67 & 04.25 & 06.40 & 20.96 \\
\hline
\end{tabular}

Table 1. Recall, precision and $f 1$ values comparison, in percentage. We compare results to those of Bruni [2] and Gullu [4]. We also compare the results of our previous work [8] on spatial line scratch detection with those after temporal filtering.

with

$$
\tilde{A}_{r, t}:=\tilde{A}_{t-1, t} \tilde{A}_{t-2, t-1} \ldots \tilde{A}_{r, r+1} .
$$

We realign each segment $S$ with respect to a reference frame $r$, which yields a realigned binary map $I_{T}^{\prime}$ defined as :

$$
I_{T}^{\prime}(x, t)= \begin{cases}1 & \text { if } \exists S \mid \tilde{x}^{r}(S)=x, \quad t(S)=t \\ 0 & \text { otherwise }\end{cases}
$$

where $\tilde{x}^{r}(S)$ is the average column index of the segment warped to the reference frame. Note that in $I_{T}^{\prime}$, a point $(x, t)$ whose value is 1 may have several corresponding original segments in the frame $t$. The trajectories of false alarms should appear in $I_{T}^{\prime}$ as vertical lines (see Figure 1(b)).

We determine the scratch trajectories using the line segment detection algorithm presented in [8]. This algorithm is well adapted to the current task, since it is designed for binary images. It also guarantees a precise and unique description of the trajectories, using the maximality and exclusion principles as defined in [9]. These properties are highly desirable here, since they avoid the task of having to bundle several partial trajectories together. Before detecting the trajectories, we perform a horizontal morphological dilation, with a distance $\tau_{x}$, on $I_{T}^{\prime}(x, t)$. This is necessary, since the spatio-temporal trajectories shown in Figure 1(c) are discretised, and therefore the trajectories may not be precisely detected without dilation. In our experiments, we set the $\tau_{x}$ parameter to 3 pixels.

Let us define a trajectory set $T$ as a set of segments whose $\mathrm{x}-\mathrm{t}$ positions are within a horizontal distance of $\tau_{x}$ of a the x-t positions of a given trajectory. We note here that the exclusion principle of the scratch detection from [8] implies that each point in $I_{t}^{\prime}$ may belong to at most one trajectory.

Once we have determined the trajectory sets, we decide whether to reject the segments belonging to them. This decision should be based on the behaviour of the global motion in the frames spanned by the trajectory set : if the scene has hardly moved, then we cannot distinguish between a true and a false scratch, and a decision should not be made. For example, in Figure 1 (c), two short trajectories (in red) which correspond to true scratches are detected, but not rejected, since there is no significant motion at that point in the video.

We take this decision by determining the maximum horizontal motion of the scene during the trajectory's timespan. To do this, we inspect the original positions of the segments and reject the trajectory set if, for any two segments $Q$ and $R$ belonging to $T$, we have :

$$
|\tilde{x}(Q)-\tilde{x}(R)| \geq \tau_{m},
$$

where $\tau_{m}$ is a motion threshold. Since the segments $Q$ and $R$ are considered to belong to the scene (apart from the case of no scene motion), this criterion reflects the maximum accumulated motion of the scene throughout the trajectory. In all of our evaluations, we set the parameter $\tau_{m}$ to 10 pixels. 


\subsection{Further filtering condition}

Apart from the motion coherency criterion, we also stipulate that trajectories should not start or stop near a scene cut. To enforce this, we simply reject the segments belonging to any trajectory set whose beginning and ending frame indices are within a temporal distance $\tau_{c}$ from a scene cut. We set $\tau_{c}$ to 4 frames. We used a simple Edge Change Ratio based scene cut detection algorithm (see [13]) for the detection of the scene cuts. This can be replaced by other scene cut detection algorithms.

\section{RESULTS}

We now present a quantitative evaluation of our algorithm in terms of recall, precision and $f 1$-score. We compare our algorithm to both the classical spatial detection algorithm of Bruni et al. [6] and the most recent temporal filtering algorithm of Gullu et al. [4]. We also compare the proposed algorithm with our previous work on spatial scratch detection [8] to ensure that our algorithm induces no degradation of results. Note that in the evaluation of our work, we impose a minimum scratch length of one tenth of the video height for all of our spatial detections.

Bruni's algorithm requires a single parameter (the scratch colour) which was set manually for each sequence. Gullu's algorithm, which uses Bruni's work for initial detection, requires two extra parameters apart from the scratch colour. The first parameter is a maximum search distance for a block matching algorithm. We chose a maximum distance of 7 pixels either side, which reflects the maximum motion we expect in the sequence. Secondly, we need a maximum mean absolute difference (MAD) threshold which identifies the presence of a scratch. We chose 15 grey levels for this threshold. These two thresholds are unspecified in [4].

We evaluate our algorithm using seven annotated videos. These were annotated by hand by defining the beginning and end points of each scratch. The videos may be found at : http://www.enst.fr/ gousseau/scratchfiltering, as well as all the results and annotations. The first three videos are very common in the scratch detection literature [14], but are of limited interest for temporal filtering, as they are short or contain no motion. The last four come from the internet archive (http://www.archive.org), or courtesy of Alvaro Pardo.

Recall is the number of correct detections divided by the total number of detections. The algorithm we use for spatial detection [8] provides a precise spatial description of the scratches. Therefore, we shall evaluate our algorithm on a pixel-wise basis, that is to say the number of correct detected pixels divided by the total number of correct pixels.

Gullu's algorithm, on the other hand, uses Bruni's spatial detection algorithm which only provides a column index to describe a scratch. Therefore, for recall purposes, we consider that the detected pixels are all those in the detected column.
In terms of precision, we must evaluate our algorithm and that of [4] differently. Since the output of Gullu's algorithm is a list of detected columns in each frame, it would be unfair to judge that algorithm on a pixel-wise basis. Therefore, we consider a detection to be correct if it touches at least one annotated scratch pixel. For our algorithm, we evaluate precision on a pixel-wise basis. Note that our evaluation confers a great advantage on Bruni's and Gullu's algorithms.

For evaluation, a detected pixel is "correct" if it is within a horizontal distance of two pixels from an annotated pixel.

Figure 1 presents a visual result of our algorithm. The false alarms due to ropes on the boat are correctly eliminated. Table 3.1 shows the quantitative comparisons.

Our algorithm obtains better $f 1$ scores on all of the examples except "Star". On the "Star" example, we are at a great disadvantage in terms of precision, as many partial scratches are present, and the algorithms of $[6,4]$ only have to touch such scratches once to obtain a correct detection.

Gullu's algorithm may induce some regression in terms of recall, and improves the precision only slightly. This performance may be explained by the extreme sensitivity to outliers in a scratch trajectory: it is sufficient that $a$ single MAD value be quite high to consider a trajectory to be a false alarm.

In comparison to our previous work [8], our temporal filtering reduces recall by a very slight amount, while precision generally improves greatly. For the first three examples, there is little temporal information and therefore very little to gain in precision. On the last four sequences, however, we may see that precision increases by up to 22 percent.

\section{CONCLUSION}

In this paper we have proposed a new approach to the temporal filtering of line scratch detections in films. The algorithm is based on the observation that false detections due to vertical scene structures move with the surrounding scene, and can therefore be identified and rejected. Robust estimation of dominant affine scene motion simplifies the problem of determining scratch trajectories to a vertical line segment detection problem. The proposed temporal filtering algorithm reduces recall only slightly, while greatly improving precision when temporal information is available. We have compared our algorithm with previous work in terms of recall, precision and $f 1$-score, and it shows considerably improved performance.

\section{ACKNOWLEDGMENTS}

The authors would like to thank Vittoria Bruni and Domenico Vitulano. The authors also acknowledge the internet archive for the content which is available online (http://www.archive.org). The authors express their thanks to Alvaro Pardo and Julieta Keldjian, for the "Keldjian" sequence. Finally, the authors thank Anil Kokaram for his image sequences, which may be found in [14]. 


\section{REFERENCES}

[1] A. Kokaram, "Detection and removal of line scratches in degraded motion picture sequences," Signal Processing VIII, vol. 1, no. 9, 1996.

[2] V. Bruni and D. Vitulano, "Fast removal of line scratches in old movies," ICPR, vol. 4, pp. 827-830, 2004.

[3] L. Joyeux, S. Boukir, and B. Besserer, "Tracking and map reconstruction of line scratches in degraded motion pictures," Machine Vision and Applications, vol. 13, no. 3, pp. 119-128, 2002.

[4] M. Gullu, O. Urhan, and S. Erturk, "Scratch detection via temporal coherency analysis and removal using edge priority based interpolation," ICAS, 2006.

[5] V. Bruni, D. Vitulano, and A. Kokaram, "Line scratches detection and restoration via light diffraction," IPSA, vol. 1, pp. 5-10, 2003.

[6] V. Bruni and D. Vitulano, "A generalised model for scratch detection," IEEE Trans on Image Proc., vol. 13, no. 1, pp. 44-50, 2004.

[7] K. Chishima and K. Arakawa, "A method of scratch removal from old movie film using variant window by hough transform," ISCIT, pp. 1559-1563, 2009.

[8] A. Newson, P. Perez, A. Almansa, and Y. Gousseau, "Adaptive line scratch detection in degraded films," CVMP, pp. 66-74, 2012.

[9] A. Desolneux, L. Moisan, and J-M. Morel, From Gestalt Theory to Image Analysis : a Probabilistic Approach, Springer-Verlag, 2008.

[10] L. Joyeux, O. Buisson, B. Besserer, and S. Boukir, "Detection and removal of line scratches in motion picture films," CVPR, vol. 1, 1999.

[11] L. Joyeux, S. Boukir, and B. Besserer, "Film line scratch removal using kalman filtering and bayesian restoration," WACV, pp. 8-13, 2000.

[12] J.M. Odobez and P. Bouthemy, "Robust multiresolution estimation of parametric motion models," International Journal of Visual Communication and Image Representation, vol. 6, pp. 348-365, 1995.

[13] R. Lienhart, "Reliable transition detection in videos: A survey and practitioner's guide," Int. Journal Image Graphics, vol. 1, no. 3, pp. 469-486, 2001.

[14] A. Kokaram, Motion Picture Restoration: Digital Algorithms for Artefact Suppression in Degraded Motion Picture Film and Video, Springer Verlag, 2001. 